\title{
BRAIN DIFFUSIVITY IN INFANTS WITH HYPOXIC ISCHEMIC ENCEPHALOPATHY FOLLOWING WHOLE BODY HYPOTHERMIA: PRELIMINARY RESULTS
}

I. Berger ${ }^{1,2}$, R. Marom ${ }^{1}$, M. Arzi ${ }^{3}$, L. Ben Sira ${ }^{3}$, H. Bassan ${ }^{2,4}$, Y. Leitner ${ }^{4}$, V. Gross-Tsur ${ }^{5}$, R. Geva ${ }^{6}$, D. Ben Bashat $^{3}$

${ }^{1}$ Neonatology, Tel Aviv Medical Center, ${ }^{2}$ Sackler Faculty, Tel Aviv University, ${ }^{3}$ The Functional Brain Center, The Wohl Institute for Advanced Imaging, ${ }^{4}$ Pediatric Neurology and Child Development, Tel Aviv Medical Center, Tel Aviv, ${ }^{5}$ Neuropediatric Unit, Shaare-Zedek Medical Center, Jerusalem, ${ }^{6}$ Multidisciplinary Brain Research Center, Bar Ilan University, Tel Aviv, Israel

Hypoxic ischemic encephalopathy is an important cause of neuropsychological deficits. Little is known about brain diffusivity in these infants following cooling and its potential in predicting outcome.Diffusion tensor imaging was applied to three groups:

1) three infants with hypoxic ischemic encephalopathy: cooled

2) three infants with hypoxic ischemicencephalopathy: non-cooled and

3) four controls. Diffusivity values at the corticospinal tract, thalamus and putamen were correlated with Apgar scores and early neurodevelopmental outcome.

While cooled infants exhibited lower Apgar scores than non-cooled infants, their developmental scores at a mean age of 8 months were higher.

All groups differed in their diffusivity values with the cooled infants showing better values compared to the non-cooled, correlating with early neurodevelopmental outcome.

These preliminary results indicate that diffusion tensor imaging performed at an early age in infants withoutcome andhypoxic ischemic encephalopathy may forecast and support the neuroprotective effect of hypothermia treatment. 